University of Nebraska - Lincoln

DigitalCommons@University of Nebraska - Lincoln

\title{
An Efficient Scheduling Scheme For On-Demand Lightpath Reservations In Reconfigurable WDM Optical Networks
}

\author{
Xi Yang \\ University of Nebraska-Lincoln, xyang@cse.unl.edu \\ Lu Shen \\ University of Nebraska-Lincoln, Ishen@cse.unl.edu \\ Ajay Todimala \\ University of Nebraska-Lincoln, ajayt@cse.unl.edu \\ Byrav Ramamurthy \\ University of Nebraska-Lincoln, bramamurthy2@unl.edu \\ Tom Lehman \\ University of Southern California
}

Follow this and additional works at: https://digitalcommons.unl.edu/cseconfwork

Part of the Computer Sciences Commons

Yang, Xi; Shen, Lu; Todimala, Ajay; Ramamurthy, Byrav; and Lehman, Tom, "An Efficient Scheduling Scheme For On-Demand Lightpath Reservations In Reconfigurable WDM Optical Networks" (2006). CSE Conference and Workshop Papers. 114.

https://digitalcommons.unl.edu/cseconfwork/114

This Article is brought to you for free and open access by the Computer Science and Engineering, Department of at DigitalCommons@University of Nebraska - Lincoln. It has been accepted for inclusion in CSE Conference and Workshop Papers by an authorized administrator of DigitalCommons@University of Nebraska - Lincoln. 


\title{
An efficient scheduling scheme for on-demand lightpath reservations in reconfigurable WDM optical networks
}

\author{
Xi Yang ${ }^{l}$, Lu Shen ${ }^{2}$, Ajay Todimala ${ }^{2}$, Byrav Ramamurthy ${ }^{2}$, Tom Lehman ${ }^{l}$ \\ ${ }^{1}$ Information Sciences Institute \\ University of Southern California \\ 3811 Fairfax Drive, Suite 200, Arlington, VA 22203 \\ Tel: (703)812-3701; Email: \{xyang, tlehman\}@isi.edu \\ ${ }^{2}$ Department of Computer Science and Engineering \\ University of Nebraska-Lincoln \\ 256 Avery Hall, Lincoln, NE 68588-0115
}

Abstract: We propose an efficient scheduling scheme that optimizes advance-reserved lightpath services in reconfigurable WDM networks. A re-optimization approach is devised to reallocate network resources for dynamic service demands while keeping determined schedule unchanged.

(C2006 Optical Society of America

OCIS codes: (060.4250) Networks; (060.4510) Optical communications

\section{Introduction}

Optical-layer reconfigurability in the next-generation wavelength-division multiplexing (WDM) networks allows bandwidth-hungry applications, such as those using Grid technologies, to share network infrastructure in an ondemand manner. Dynamic demands by such applications could considerably compromise the efficiency of conventional network operations that assume static or predictable demands. In addition, many applications, e.g., real-time e-science experiments, need guaranteed lightpath connections during a specified period of time in future. The application users prefer to make advance reservations for end-to-end lightpaths for predefined service durations where the starting time of the lightpath demand can be days to weeks into the future. Such an advance reservation of a lightpath is called scheduling of a lightpath and correspondingly the lightpath itself is termed as a scheduled lightpath demand (SLD) [1][2]. Almost all previous studies considered static scheduling, in which the whole set of SLDs are known before any actual provisioning happens in the network. In this study, we address fully dynamic scheduling of SLDs (D-SLDs), whose precise arrival time is unknown and unpredictable.

Dynamic SLDs (D-SLDs) can be classified into two main types. We use two example applications to characterize them respectively. The first is to schedule a real-time, collaborative scientific experiment. A lightpath is scheduled between the data collection and data processing facilities at a fixed startup time for a fixed duration, e.g., 9:0010:00AM on Monday. We term such a demand to be of the time-fixed type. In the second example, a financial institution demands a scheduled lightpath on a weekly basis to backup its huge database to a data depot facility in a different city. The transfer takes up to one hour. It specifies a loose starting time window, say 1:00-5:00AM on Saturdays, during which any lightpath starting time is acceptable. We term such a demand to be of the time-window type. Static time-window SLDs are modeled as sliding scheduled traffic in [3].

Generally, both time-fixed and time-window D-SLDs need deterministic services. By a deterministic service, we mean that after submitting a request for a lightpath, a user expects a deterministic answer on whether the request can be accommodated and if so, the precise schedule information for the request. Even for a time-window D-SLD, a deterministic schedule should be returned so that the requestor knows the exact starting time for transferring the application data. Deterministic service provisioning in the presence of D-SLDs may be inefficient in terms of network resource utilization. Unlike static demands, D-SLDs cannot be precisely predicted and thus resource allocation for D-SLDs is difficult to be optimized as a whole. Though the deterministic answer obtained for a DSLD can be optimal at the current time, the network may still become sub-optimal with the arrivals of future DSLDs. Interestingly, before a D-SLD is physically provisioned in the network, any adjustment of the resources reserved for this D-SLD, e.g., rerouting and reassignment of wavelength, will not disrupt its service. Therefore, we have the opportunity to perform re-optimization for all D-SLDs scheduled to be carried in the future.

In this study, we propose that resources reserved for scheduled lightpaths be re-optimized before they are physically provisioned so that better network performance can be achieved. We consider both time-fixed and time-window DSLDs. Our scheme will provide a deterministic scheduling algorithm for D-SLDs and re-optimization techniques for the scheduled lightpaths that have not been physically provisioned. In so doing it manages to reduce by up to a half the performance gap between the predefined static SLDs and the unpredictable dynamic SLDs.

\section{Network Model, problem statement and our solution scheme}

We consider WDM wavelength-routed mesh networks, where a set of reconfigurable optical cross-connects (OXCs) are interconnected by optical fibers. To facilitate lightpath scheduling, the network time is slotted [4]. A time slot is 


\section{OTuN2.pdf}

a minimum time unit in the network, each having an equal and fixed length. The wavelength availability in a time slot is independent of its availability in other time slots. As a result, a wavelength can be reused over different links as well as multiple time slots. We assume the network is under wavelength continuity constraint.

A D-SLD is denoted by a 5-tuple, $(s, d, t, e, x)$, where $s$ and $d$ denote source and destination nodes of the demand, $t$ and $e$ denote the starting time slot and duration of the demand respectively, and $x$ is the maximum lightpath length in order to confine the optical impairs and end-to-end delay. The time-fixed D-SLD has fixed starting time, while the starting time of the time-window demand can be any value in a range of contiguous time slots. However once a time-window D-SLD is scheduled, its starting time cannot be altered. A lightpath admitted in the network is in either a scheduled state or an in-service state. Scheduled state indicates that its starting time, routing and wavelength assignment have been determined, but the lightpath has not been physically provisioned. In-service state implies that the lightpath is currently being used for data transmission. Figure 1 shows an example of lightpath reservation system. The horizontal axis represents the time line and the vertical line denotes different wavelengths.

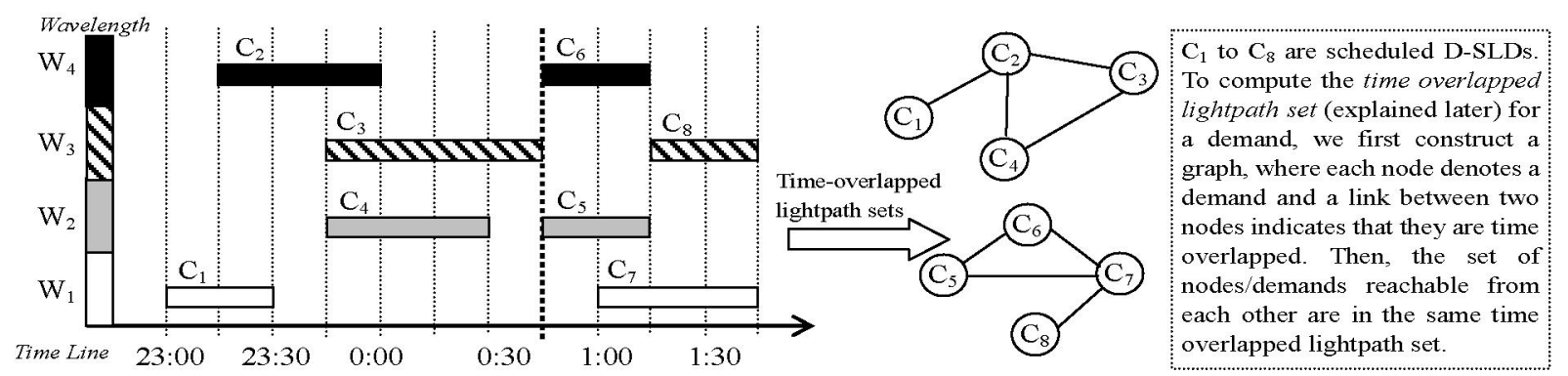

Figure 1 An example of lightpath reservation system.

Given a network topology, the wavelength availability information in each time slot, and a D-SLD request, the dynamic lightpath scheduling problem is to determine the starting time for the D-SLD and assign a route and wavelength (RWA) for the lightpath with the objective of reducing the network blocking probability.

We propose a two-phase approach to schedule a D-SLD. In phase I, our approach schedules the starting time and conducts RWA for the D-SLD with a load-balancing objective. The load balancing objective is expressed as: minimizing $\underset{t \leq i \leq t+e-1, l \in L}{\operatorname{MaX}} u_{l}^{i}$, where $L$ is the set of links in the network, and $t$ and $e$ are the starting time and duration of the D-SLD respectively. $u_{l}^{i}$ is the number of used wavelengths on the link $l$ in time slot $i$. Given this D-SLD, we first compute k-shortest paths between its source and destination whose lengths are not greater than the maximum path length $(x)$ of the demand. These paths are used as candidate routes for the demand. We use a slotted first-fit (SFF) scheme for wavelength assignment. Given a route, $S F F$ picks the first common wavelength that is available on every link on the path during all the time slots between its starting and ending time. After computing the load balancing objective values iteratively on each candidate path over each possible starting time slot, our approach chooses the solution that minimizes the load balancing objective value. If the D-SLD is blocked in phase I, our approach enters phase II where the re-optimization procedure starts.

The objective of our approach in phase II is to eliminate the blocking generated in phase I. This is achievable because we can re-provision those scheduled lightpaths for re-optimization. The re-provisioning is risk-free since they are not in-service yet. First, the re-optimization procedure finds the time-overlapped lightpath set of the blocked demand. The time-overlapped lightpath set is a set containing all the lightpaths correlated to each other in terms of their service time (Figure 1 explains how to compute the time-overlapped lightpath set). Second, the re-optimization procedure sorts all the demands in the time-overlapped lightpath set according to a specific ordering scheme (as explained in next paragraph). Third, it releases all the lightpaths in the set and tries to re-provision them (including the blocked demand) one by one in the sorted order with our load-balancing objective. If all the demands are provisioned successfully, the blocked is removed. Otherwise, the blocked demand in Phase I remains blocked and we restore the existing lightpaths to their original states.

The ordering scheme used in the re-optimization procedure of Phase II has a significant effect for our approach. We use a combination of multiple keys for ordering. Given a set of scheduled lightpaths, we first sort them according to the non-decreasing order of their starting time. If two lightpaths have the same starting times, we break the tie by sorting them according to the non-decreasing order of the number of hops on their minimum-hop paths. If they are still tied, we break the tie by sorting them according to the non-decreasing order of their service durations. 


\section{OTuN2.pdf}

Therefore, the lightpaths are sorted according to the rules of the earliest starting time first, maximum hop first, and longest duration first with decreasing priorities.

\section{Illustrative numerical results}

In this section, we present numerical results of our proposed approach for on-demand lightpath reservation. We conduct simulation experiments on a 24-node, 84 link network. The duration of each time slot is set to 15 minutes. In each simulation we simulate 10,000 D-SLDs consisting of a mix ratio of 7:3 of time-fixed and time-window demands. The window size of the time-window demands is uniformly distributed in the range $[5,50]$ minutes. The duration of D-SLDs is measured in number of time slots and is a weighted non-uniform distribution in the range [1, 100]. The inter-arrival time and the starting times of D-SLDs are in Poisson distributions. We use two metrics for comparison, blocking probability (BP) and service blocking probability (SBP). The service blocking probability is measured as the ratio of the sum of the durations of blocked D-SLDs to the sum of the durations of all the D-SLDs. Because D-SLDs may have different durations, SBP provides a fair measurement on the network performance. We conduct our experiments in the cases with $4,8,16$, and 32 wavelengths. The path length constraint is set to $600 \mathrm{~km}$, which is considered as a typical reach distance of all-optical signals.

Figure 2 compares the performance, in terms of BP and SBP, of lightpath scheduling approaches with and without re-optimization in the networks with 32 wavelengths. Other cases achieve similar results. As shown in the figure, the approach with re-optimization reduces both the blocking probability and the service blocking probability significantly. On average, compared to the approach without re-optimization, our approach eliminates $33 \%, 53 \%$, $52 \%$, and $51 \%$ blockings in phase II for different cases. Figure 3 compares the service blocking probability of the dynamic scheduling approaches with a static approach for the case of 16 wavelengths. In the static approach, all the lightpath demands are known in advance so that its solution may be used as a lower bound for comparison. Figure 3 shows a large performance gap for the solutions between the dynamic without re-optimization and the static approach in terms of SBP. It also shows that our re-optimization approach reduces the performance gap by a large margin. This improvement arises from re-provisioning those scheduled lightpaths before they are placed in service. However, it should be noted that it is impossible to remove this gap completely because the dynamic approach can never precisely predict the traffic pattern in the future. Also, due to the requirement for deterministic services, the starting time of any scheduled D-SLD cannot be changed, which leaves a much smaller space for re-optimization.

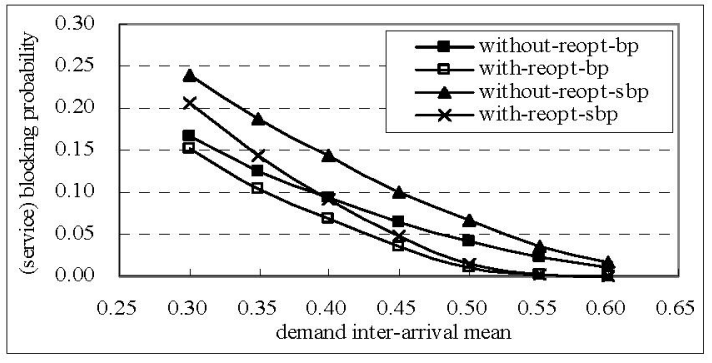

Figure 2 Results with and without re-optimization at blocking in the network with 32 wavelengths.

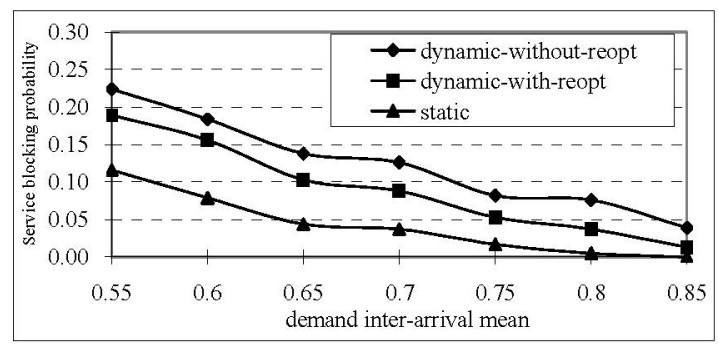

Figure 3 Comparison of SBP of dynamic scheduling with and without re-optimization and static scheduling.

\section{Conclusion}

In this study we addressed on-demand advance reservations for lightpath services. We considered both time-fixed and time-window D-SLDs. Compared to predefined static demands, the unpredictable dynamic demands have a very limited potential for optimization. Therefore, we proposed an efficient two-phase lightpath scheduling scheme to reduce up to a half of the performance gap between fully static and fully dynamic SLDs. Our simulation experiments showed that $33 \%-53 \%$ of blockings can be eliminated at admission. This is a great performance improvement for those network users who require both dynamic and deterministic scheduled lightpath services.

\section{References}

[1]. S. Figueira, N. Kaushik and S. Naiksatam, "Advance reservation of lightpaths in optical-network based Grids," in Proc. of IEEE BroadNets/GridNets 2004, San Jose, CA, USA, Oct. 2004.

[2]. J. Kuri et al., "Routing and wavelength assignment of scheduled lightpath demands", IEEE Journal of Selected Areas in Communications (JSAC), vol. 21, no. 8, pp. 1231-1240, Oct. 2003.

[3]. B. Wang, T. Li, X. Lou, Y. Fan, and C. Xin, "On service provisioning under a scheduled traffic model in reconfigurable WDM optical networks," in Proc. of IEEE BroadNets' '05, Boston, MA, USA, Oct. 2005.

[4]. A. Maach and A. Hafid, "Resources reservation in advance in slotted optical networks," in Proc. of the Third International Conference on Optical Communications and Networks (ICOCN 2004), Hong Kong, China, Nov. 2004. 